International Journal of Biomathematics

Vol. 5, No. 6 (November 2012) 1299001 (6 pages)

(C) World Scientific Publishing Company

DOI: $10.1142 / \mathrm{S} 179352451299001 \mathrm{X}$

\title{
AUTHOR INDEX (Volume 5)
}

Agarwal, M. \&

Bhadauria, A. S., Modeling H1N1 flu epidemic with contact tracing and quarantine

Ali, S., see Rich, S.

Arghirescu, S., see

Precup, R.

Bhadauria, A. S., see Agarwal, M.

Bhatia, S. S., see Kaur, N.

Bhunu, C. P., see Mushayabasa, S.

Bogaert, J., see Lin, H.

$\mathrm{Bu}, \mathrm{L}$, see Lou, J.

Cai, Y., Wang, W. \& Wang, J., Dynamics of a diffusive predator-prey model with additive Allee effect

Calkins, G. W., see Rich, S.

Cao, H., Xiao, Y. \&

Zhou, Y., The

dynamics of a discrete SEIT model with age and infection age structures

Chen, F., see Li, Z.

Chen, H., see Wang, Q.

Chen, L. \& Ma, W., A nonlinear delay model describing the growth of tumor cells under immune surveillance against cancer and its stability analysis

Chen, L., see Dai, C.
2 (2012) 1250023

3 (2012) 1260017

5 (2012) 1250038

6 (2012) 1250053

2 (2012) 1250026

5 (2012) 1250038

5 (2012) 1250040

4 (2012) 1250029

3 (2012) 1260009

5 (2012) 1250032

6 (2012) 1250053

3 (2012) 1260004

6 (2012) 1250057

3 (2012) 1260010

6 (2012) 1250059
Chen, L., see Zhang, Y.

Cucuianu, A., see

Precup, R.

3 (2012) 1260005

2 (2012) 1250026

2 (2012) 1250024

4 (2012) 1250037

Cui, J.-A., see Qi, L.

Cui, J.-A., see Wei, J.

3 (2012) 1260003

4 (2012) 1250014

Dai, C., Zhao, M. \&

Chen, L., Homoclinic

bifurcation in

semi-continuous

dynamic systems

Davvaz, B., see Ghadiri, M.

Deng, C. \& Gao, H., Stability of SVIR system with random perturbations

4 (2012) 1250025

Devi, S., Nonconstant prey harvesting in ratio-dependent predator-prey system incorporating a constant prey refuge

Ding, Y., see Gao, S.

Du, Y. \& Xu, R., Traveling wave solutions in a three-species food-chain model with diffusion and delays

Enatsu, Y., see Nakata, Y.

1 (2012) 1250002

1 (2012) 1250009

Englund, G., see Liao, J. $\quad 5$ (2012) 1250048

Feng, E., see Shen, L. 2 (2012) 1250013

Feng, E., see Wang, L. 4 (2012) 1250034

Fu, S., see Zhang, L.

$\mathrm{Fu}, \mathrm{X}$., see Wu, Q.

Gakkhar, S., Singh, A. \& Singh, B. P., Effects of delay and seasonality on toxin producing
6 (2012) 1250052

1 (2012) 1250004 
Phytoplankton-

Zooplankton system

5 (2012) 1250047

Gámez, M., see López, I.

Gámez, M., see Molnár, $\mathrm{S}$.

Gao, H., see Deng, C.

Gao, S., Ding, Y. \& Xie, J., Role of disease propagation in migratory bird population

Gao, X., see Liu, L.

Gao, Y., see Qi, L.

Garay, J., see López, I.

Ghadiri, M., Davvaz, B. \& Nekouian, R.,

$H_{v}$-semigroup

structure on

$F_{2}$-offspring of a gene pool

Ghosh, M., see Kaur, N.

Ghosh, M., see Qian, D.-L.

Gong, Z., see Wang, L.

Goulionis, J. E.,

Switching and sequencing available therapies so as to maximize a patient's expected total lifetime

Gray, R. R., see Prosperi, M. C. F.

Guo, Z., see Jiang, Z.

Han, E., see Lou, J.

Han, M., see Li, Z.

Hota, M. K. \&

Srivastava, V. K., Multistage filters for identification of eukaryotic protein coding regions

Hu, G.-P. \& Li, X.-L., Turing patterns of $a$ predator-prey model with a modified Leslie-Gower term and cross-diffusions

$\mathrm{Hu}, \mathrm{P}$, see Zhang, L.

Huang, J., see Liu, C.

Huo, H. F., see Meng, X. Y.

Irie, H. \& Tokita, K., Species-area

3 (2012) 1260002

4 (2012) 1250031

4 (2012) 1250037

6 (2012) 1250054 relationship for

power-law species

abundance

distribution

3 (2012) 1260014

Ji, C. \& Jiang, D.,

Dynamics of an $H I V-1$

infection model with

cell-mediated immune

response and stochastic perturbation

5 (2012) 1250039

5 (2012) 1250039

Jiang, D., see Ji, C.

Jiang, W., see Wang, J.

Jiang, Z., Guo, Z. \& Sun, Y., Stability analysis of
5 (2012) 1250040

3 (2012) 1260016

4 (2012) 1250034

4 (2012) 1250028

6 (2012) 1250055

1 (2012) 1250007

5 (2012) 1250032

6 (2012) 1250057

2 (2012) 1250018

6 (2012) 1250060

6 (2012) 1250052

2 (2012) 1250010

1 (2012) 1250008 a predator-prey model

Kang, B., see Liu, B.

Kaur, N., Ghosh, M. \&

Bhatia, S. S., Modeling

the spread of $H I V$ in a

stage structured

population: Effect of awareness

5 (2012) 1250040

Li, C. \& Tang, S., The effects of timing of pulse spraying and releasing periods on dynamics of generalized predator-prey model

Li, W., see Liu, Y.

Li, W.-T., see Zhang, J.-F.

Li, X. \& Qu, R.,

Parameter

identification and terminal steady-state optimization for $S$ system in microbial continuous fermentation

2 (2012) 1250020

Li, X.-L., see Hu, G.-P. Li, X.-Z., see Qian, D.-L. Li, Y. \& Zhang, T., Almost periodic solution for a discrete Hematopoiesis model with time delay

1 (2012) 1250007 3 (2012) 1260006

1 (2012) 1250012 5 (2012) 1250044

6 (2012) 1250049

3 (2012) 1260016
Li, Z. \& Xu, R.,

Traveling waves in a reaction-diffusion predator-prey system with nonlocal delays

Li, Z., Han, M. \& Chen, F., Global stability of a stage-structured 
predator-prey model with modified

Leslie-Gower and

Holling-type II

schemes

6 (2012) 1250057

Li, Z., see Liao, J.

Li, Z., see Lin, H.

Liang, J.-H., see Tan, Y.-S.

Liang, T., see Lin, H.

Liao, J., Lu, H., Li, Z., Meng, X. \& Englund, G., The dynamical models of activated sludge system:

Stochastic cellular automaton and differential equations

Lin, H., Zhao, J., Liang, T., Bogaert, J. \& Li,

Z., A classification indices-based model for Net Primary

Productivity (NPP)

and potential

productivity of vegetation in China

Liu, B., Tian, Y. \&

Kang, B., Dynamics

on a Holling II

predator-prey model with state-dependent impulsive control

Liu, C., Zhang, Q., Huang, J. \& Tang, W., Dynamical analysis and control in a delayed differential-algebraic bio-economic model with stage structure and diffusion

Liu, J., Zhou, H. \& Zhang, L., Cross-diffusion induced Turing patterns in a sex-structured predator-prey model

Liu, L. \& Gao, X., Qualitative study for a multi-drug resistant TB model with exogenous reinfection and relapse
5 (2012) 1250048

3 (2012) 1260009

3 (2012) 1260018

3 (2012) 1260009

5 (2012) 1250048

3 (2012) 1260009

3 (2012) 1260006

2 (2012) 1250010

4 (2012) 1250016

4 (2012) 1250031
Liu, M., see Lv, J.

5 (2012) 1250035

Liu, S., Xie, X. \& Tang,

J., Competing population model with nonlinear intraspecific regulation and maturation delays

Liu, S., see Wang, J.

Liu, Y., Li, W. \& Ma, R., Particle swarm optimization on flexible docking

Liu, Y.-P. \& Luo, Z.-X., Persistence, extinction and stability for nonlinear plankton allelopathy model with delayed negative feedbacks

López, I., Standovár, T., Garay, J., Varga, Z. \& Gámez, M., Statistical detection of spatial plant patterns under the effect of forest use López, I., see Molnár, S. Lou, J., Bu, L., Han, E., Ruan, Y., Xing, H. \& Shao, Y., Modeling primary and secondary drug resistances under China's

"four-free-one-care policy"

Lu, H., see Liao, J.

Luo, Y.-J., see Luo, Z.-X.

Luo, Z.-X., Yang, J.-Y. \& Luo, Y.-J., Optimal control for a nonlinear n-dimensional competing system with age-structure

3 (2012) 1260007

3 (2012) 1260001

5 (2012) 1250044

2 (2012) 1250027

6 (2012) 1250054

6 (2012) 1250051

5 (2012) 1250032

5 (2012) 1250048

3 (2012) 1260008

3 (2012) 1260008

Luo, Z.-X., see Liu, Y.-P.

2 (2012) 1250027

Lv, J., Wang, K. \& Liu, M., Dynamical properties of a stochastic two-species Schoener's competitive model

5 (2012) 1250035

Ma, R., see Liu, Y.

Ma, S. \& Zu, Y., Stability analysis of a discrete nonlinear
5 (2012) 1250044 
delay survival red blood cells model

4 (2012) 1250030

Ma, W., see Chen, L.

Meng, X. Y., Huo, H. F. \& Zhang, X. B., The effects of harvesting and time delay on predator-prey systems with

Beddington-Deangelis functional response

Meng, X., see Liao, J.

Michaelson, J. S., see Rich, S.

Mishra, S., Verma, N. K. \& Siddiqui, S. U., A suspension model for blood flow through a catheterized artery

Misra, J. C., see Sinha, A.

Molnár, S., Gámez, M. \& López, I., Observation of nonlinear verticum-type systems applied to ecological monitoring

Mulone, G. \& Straughan, B., Modeling binge drinking

Muroya, Y., see Nakata, Y.

Mushayabasa, S. \& Bhunu, C. P., Modeling the impact of voluntary testing and treatment on tuberculosis transmission dynamics

Nakata, Y., Enatsu, Y. \& Muroya, Y., Two types of condition for the global stability of delayed SIS epidemic models with nonlinear birth rate and disease induced death rate

Nekouian, R., see Ghadiri, M.

Petrauskas, E., see Rupšys, P.

Precup, R., Arghirescu, S., Cucuianu, A. \& Şerban, M., Mathematical modeling

6 (2012) 1250053

5 (2012) 1250033

5 (2012) 1250042

6 (2012) 1250051

1 (2012) 1250005

1 (2012) 1250009

4 (2012) 1250029

1 (2012) 1250009

4 (2012) 1250011

5 (2012) 1250045 of cell dynamics after allogeneic bone

marrow

transplantation

Prosperi, L., see

Prosperi, M. C. F.

Prosperi, M. C. F.,

Prosperi, L., Gray, R.

R. \& Salemi, M., On

counting the frequency distribution of string motifs in molecular sequences

6 (2012) 1250055

Qi, L. \& Cui, J.-A., The delayed Barbour's model for schistosomiasis

Qi, L., Cui, J.-A., Gao, Y. \& Zhu, H., Modeling the schistosomiasis on the islets in Nanjing

Qian, D.-L., Li, X.-Z. \& Ghosh, M., Coexistence of the strains induced by mutation

Qu, R., see Li, X.

Radhakrishnamacharya, G., see Ramana Kumari, A. V.

2 (2012) 1250026

6 (2012) 1250055

2 (2012) 1250024

4 (2012) 1250037

3 (2012) 1260016

2 (2012) 1250020

6 (2012) 1250015

Ramana Kumari, A. V. \& Radhakrishnamacharya, G., Effect of slip and magnetic field on peristaltic flow in an inclined channel with wall effects

Ran, P., see Zhu, W.

Razvan, M. R. \&

Yasaman, S., Global

dynamics of a

differential susceptibility model

6 (2012) 1250015

4 (2012) 1250022

Rich, S., Ali, S., Calkins, G. W. \& Michaelson, in childhood hematological malignancies

Ruan, Y., see Lou, J.

Rupšys, P. \& Petrauskas, E., Analysis of height curves by stochastic differential equations 
Saitoh, K., see Shoji, H.

Salemi, M., see Prosperi, M. C. F.

Şerban, M., see Precup, R.

Shao, Y., see Lou, J.

Shen, L., Feng, E. \& Wu, Q., Impulsive control in microorganisms continuous fermentation

Shi, W.-W. \& Tan, Y.-S., Transmission dynamics and optimal control of an influenza model with quarantine and treatment

3 (2012) 1260013

6 (2012) 1250055

2 (2012) 1250026

5 (2012) 1250032

2 (2012) 1250013

3 (2012) 1260011

Shoji, H. \& Saitoh, K., Pattern formation in chemotaxic reaction-diffusion systems

Siddiqui, S. U., see Mishra, S.

Singh, A., see Gakkhar, S.

Singh, B. P., see Gakkhar, S.

Sinha, A. \& Misra, J. C., Influence of slip velocity on blood flow through an artery with permeable wall: A theoretical study

Srikanth, D., see Srinivasacharya, D.

Srinivasacharya, D. \& Srikanth, D., Flow of micropolar fluid through catheterized artery - $A$ mathematical model

Srivastava, V. K., see Hota, M. K.

Standovár, T., see López, I.

Straughan, B., see Mulone, G.

Sun, Y., see Jiang, Z.

Takeuchi, Y., see Wang, J.

Takeuchi, Y., see Zu, J. Tan, Y.-S., Liang, J.-H. \& Tang, S.-Y., The dynamical behavior of

3 (2012) 1260013

5 (2012) 1250033

5 (2012) 1250047

5 (2012) 1250047

5 (2012) 1250042

2 (2012) 1250019

2 (2012) 1250019

2 (2012) 1250018

6 (2012) 1250054

1 (2012) 1250005

1 (2012) 1250007

3 (2012) 1260001

3 (2012) 1260015 non-smooth system with impulsive control strategies

3 (2012) 1260018

Tan, Y.-S., see Shi, W.-W.

Tang, J., see Liu, S.

Tang, S., see Li, C.

Tang, S.-Y., see Tan, Y.-S.

Tang, W., see Liu, C.

Tian, F., see Zhu, W.

Tian, Y., see Liu, B.

Tokita, K., see Irie, H.

Varga, Z., see López, I.

Verma, N. K., see Mishra, S.

Wang, J. \& Jiang, W., Hopf bifurcation analysis of two sunflower equations

Wang, J. L., see Zu, J.

Wang, J., Takeuchi, Y. \& Liu, S., A multi-group SVEIR epidemic model with distributed delay and vaccination

Wang, J., see Cai, Y.

Wang, K., see Lv, J.

Wang, L. \& Xu, R., Mathematical analysis of an improved hepatitis $B$ virus model

3 (2012) 1260011

3 (2012) 1260007

1 (2012) 1250012

3 (2012) 1260018

2 (2012) 1250010

4 (2012) 1250022

3 (2012) 1260006

3 (2012) 1260014

6 (2012) 1250054

5 (2012) 1250033

1 (2012) 1250001

3 (2012) 1260015

3 (2012) 1260001

2 (2012) 1250023

5 (2012) 1250035

Wang, L., Xiu, Z., Gong, Z. \& Feng, E., Modeling and parameter identification for multistage simulation of microbial bioconversion in batch culture

4 (2012) 1250034

Wang, Q., Xiao, Y., Chen, H. \& Zou, Q., Scanning and analysis of mismatch distribution on human genome

3 (2012) 1260010

Wang, S. \& Zhou, Y., Global dynamics of an in-host HIV-1 infection model with the long-lived infected cells and four intracellular delays
1 (2012) 1250006 
Wang, S. \& Zou, D., Viral dynamics in a distributed time delayed $\mathrm{HCV}$ pathogenesis model

Wang, W., see Cai, Y.

Wei, J. \& Cui, J.-A., Dynamics of SIS epidemic model with the standard incidence rate and saturated treatment function

Weng, P., see Yu, X.

$\mathrm{Wu}, \mathrm{C}$., see $\mathrm{Yu}, \mathrm{X}$.

Wu, Q., Fu, X. \& Zhu, G., Global attractiveness of discrete-time epidemic outbreak in networks

Wu, Q., see Shen, L.

Xiao, Y., see Cao, H.

Xiao, Y., see Wang, Q.

Xiao, Y., see Zhang, J.

Xiaohong, T., Stability analysis of a delayed SIRS epidemic model with vaccination and nonlinear incidence

Xie, J., see Gao, S.

Xie, X., see Liu, S.

Xing, H., see Lou, J.

Xiu, Z., see Wang, L.

$\mathrm{Xu}, \mathrm{R}$., see Du, Y.

$\mathrm{Xu}, \mathrm{R}$., see Li, Z.

$\mathrm{Xu}, \mathrm{R}$., see Wang, L.

$\mathrm{Xu}, \mathrm{R} .$, Global dynamics of a delayed $H I V-1$ infection model with absorption and saturation infection

Yan, X.-P., see Zhang, J.-F.

Yang, J.-Y., see Luo, Z.-X.

Yasaman, S., see Razvan, M. R.

Yu, X., Wu, C. \& Weng, P., Traveling waves for a SIRS model with nonlocal diffusion

Zha, L., Cui, J.-A. \& Zhou, X.,
6 (2012) 1250056

2 (2012) 1250023

3 (2012) 1260003

5 (2012) 1250036

5 (2012) 1250036

1 (2012) 1250004

2 (2012) 1250013

3 (2012) 1260004

3 (2012) 1260010

4 (2012) 1250017

6 (2012) 1250050

3 (2012) 1260002

3 (2012) 1260007

5 (2012) 1250032

4 (2012) 1250034

1 (2012) 1250002

5 (2012) 1250043

1 (2012) 1250006

3 (2012) 1260012

6 (2012) 1250049

3 (2012) 1260008

5 (2012) 1250046

5 (2012) 1250036
Ratio-dependent predator-prey model with stage structure and time delay

4 (2012) 1250014

Zhang, J. \& Xiao, Y., Modeling strategies for controlling H1N1 outbreaks in China

Zhang, J.-F., Li, W.-T. \& Yan, X.-P.,

Bifurcation and spatiotemporal patterns in a homogeneous diffusion-competition system with delays

6 (2012) 1250049

Zhang, L., Fu, S. \& Hu, P., Effect of cross diffusion in a competition model with stage structure

6 (2012) 1250052

4 (2012) 1250016

2 (2012) 1250010

1 (2012) 1250003

Zhang, T., see Li, Y.

Zhang, X. B., see Meng, $\mathrm{X}$. $\mathrm{Y}$.

1 (2012) 1250008

Zhang, Y. \& Chen, L., The periodic Volterra model with mutual interference and impulsive effect

Zhao, J., see Lin, H.

Zhao, M., see Dai, C.

Zhou, H., see Liu, J.

Zhou, X., see Zha, L.

Zhou, Y., see Cao, H.

Zhou, Y., see Wang, S.

Zhu, G., see Wu, Q.

Zhu, H., see Qi, L.

Zhu, W., Tian, F. \& Ran, P., Analytical solutions of non-Fourier Pennes and Chen-Holmes equations

Zou, D., see Wang, S.

Zou, Q., see Wang, Q.

$\mathrm{Zu}$, J., Wang, J. L. \& Takeuchi, Y.,

Coevolutionary

dynamics of

predator-prey

interactions

$\mathrm{Zu}, \mathrm{Y}$., see Ma, S.
3 (2012) 1260005

3 (2012) 1260009

6 (2012) 1250059

4 (2012) 1250016

4 (2012) 1250014

3 (2012) 1260004

6 (2012) 1250058

1 (2012) 1250004

4 (2012) 1250037

4 (2012) 1250022

6 (2012) 1250056

3 (2012) 1260010

3 (2012) 1260015

4 (2012) 1250030 